\title{
Proteomics Based Mongolian Medicine Modified Sugmul-7 Mechanism of Regulating Endocrine Function in Hyperplasia of the Breast
}

\author{
Tong Shan ${ }^{1,2}$, Tsend Ayushi ${ }^{3}$, Tuul Khalzaibaast ${ }^{4}$, Lian Bao Chen ${ }^{5}$, Chao Lu Meng ${ }^{5}$, Xing An ${ }^{5}$, \\ Ju Hua Gong', Wang Huan ${ }^{7, *}$ \\ ${ }^{1}$ Affiliated Hospital of Inner Mongolia University for Nationalities, Tongliao, China \\ ${ }^{2}$ Mongolian National University of Medical Sciences, Ulaanbaatar, Mongolia \\ ${ }^{3}$ Mongolian Medical Research Center, International school of Mongolian Traditional Medicine, MNUMS, Ulaanbaatar, Mongolia \\ ${ }^{4}$ Traditional Medicine Department, Mongolian University Pharmaceutical Sciences, Ulaanbaatar, Mongolia \\ ${ }^{5}$ Mongolian Medicine and Pharmaceutical School of Inner Mongolia University of Nationalities, Tongliao, China \\ ${ }^{6}$ Traditional Chinese Medicine (Mongolian Medicine), Inner Mongolia University for Nationalities, Tongliao, China \\ ${ }^{7}$ Mongolian Medicine and Pharmaceutical School of Inner Mongolia University for Nationalities, Mongolian Pharmacology Laboratory, \\ Tongliao, China
}

\section{Email address:}

tongshan100@163.com (Tong Shan),tsendayush@mnums.edu.mn (T. Ayushi),khtuul2017@gmail.com (T. Khalzaibaast), 2223246638@qq.com (Lian Bao Chen),63625492@qq.com (Chao Lu Men),1911543587@qq.com (Xing An),

18747552667@163.com (Ju Hua Gong), wanghuan8217776@163.com (Wang Huan)

${ }^{*}$ Corresponding author

\section{To cite this article:}

Tong Shan, Tsend Ayushi, Tuul Khalzaibaast, Lian Bao Chen, Chao Lu Meng, Xing An, Ju Hua Gong, Wang Huan. Proteomics Based Mongolian Medicine Modified Sugmul-7 Mechanism of Regulating Endocrine Function in Hyperplasia of the Breast. Clinical Medicine Research. Vol. 9, No. 1, 2020, pp. 11-19. doi: 10.11648/j.cmr.20200901.13

Received: January 14, 2020; Accepted: February 7, 2020; Published: February 14, 2020

\begin{abstract}
The aim of this study was to explore the mechanism of regulating the endocrine function of hyperplasia breast in rats with modified Mongolian medicine Sugmul-7 by proteomics, and provide experimental basis for its development and clinical application. Ninety female SD rats were randomly divided into normal group, model group, and modified Sugmul-7 low, medium, and high doses in a total of 5 groups. After the start of the experiment, each rat except the normal group was intraperitoneally injected with estradiol $25 \mathrm{~d}$ and progesterone $6 \mathrm{~d}$ at a standard dose of $0.3 \mathrm{mg} / \mathrm{kg}$ for a total of 31 days. Four differential proteins were found through proteomic analysis, namely Ppp3cb, Cacybp, Gstz1Gstz1, and Nmd3 (and satisfying the standard conditions of 1.2 times to 0.8333 times) and found 5 related pathways. Mongolian medicine modified Sugmul-7 estradiol and progesterone-induced hyperplasia of the breast, Ppp3cb, Cacybp, Gstz1Gstz1, Nmd3 and other 4 differential proteins, through cGMP-PKG signal pathway, thyroid hormone signal pathway, estrogen signal pathway gastrointestinal signal related pathways, reproductive system related pathways, etc. regulate endocrine related pathways to treat the disease.
\end{abstract}

Keywords: Mongolian Medicine Modified Sugmul-7, Hyperplasia of the Breast, Endocrine Function, Proteomics

\section{Background}

Hyperplasia of mammary glands is a common breast disease in clinical practice. This disease occurs in young and middle-aged women aged 25-45 years. According to the results of the investigation, $[1,2]$. The pathogenesis of the disease is analyzed as: mental workers, nervousness, anxiety, dumbness, introverted personality, etc. In the future, the pace of urban life will accelerate, social pressure will increase, and the impact of fertility will increase the incidence of breast hyperplasia year by year. Other pathogenesis has not been thoroughly studied, but it is preliminarily determined that it is caused by endocrine imbalance caused by emotions and certain external or internal causes. Fan Fengying's research 
suggests that hyperplasia of the breast is associated with an increase in estradiol [3]; Lu Jingbo's research suggests that an increase in prolactin is associated with breast hyperplasia [4]; $\mathrm{Wu}$ Jieping's research shows that [5], estrogen-progestin disorders are also one of the causes of breast hyperplasia.

Modern medicine often uses surgical and non-surgical treatments for breast hyperplasia (including drug therapy-androgen, brass body, vitamin E, physical therapy, etc.), but long-term use of hormonal drugs has large side effects and interferes with hormone levels in the body. However, the curative effect is not exact; there are also surgical resection treatments, but the endocrine function cannot be fundamentally adjusted, the effect is not ideal, the recurrence rate is high and the appearance is beautiful [6]. At present, there is no widely applicable and effective therapeutic drug or scheme for breast hyperplasia [7].

Mongolian medicine clinically classifies breast hyperplasia as a category of "breast lump". It is a disease in which female breast swelling and hot pain are the main symptoms. In severe cases, suppuration and severe pain are present. Mucoidosis (one of the pathogenic factors of Mongolian medicine, including pathogenic microorganisms such as bacteria and viruses), and its blood (blood, one of the pathogenic factors of Mongolian medicine, that is, diseased blood) is the cause of "breast tumor", which is clinically sticky. The epidemic and its susceptibility (blood) are the causative factors of the disease [8]. The pathological mechanism is that the body is affected by certain pathogenic conditions (diet, living, climate, other). The dynamic balance of the "three roots and seven hormones" is disrupted, causing the "essence and dross decomposition" disorder, which causes the liver to break down its blood (bad blood). Its finally decomposed essence will cause disease (breast hyperplasia) when it is transported to various tissues and organs (breast).

In other words, the theory of Mongolian medicine believes that the pathogenesis of hyperplasia of the breast is the disorder of "essence and dross decomposition", which further results in metabolic dysfunction. During the treatment, a scheme to adjust the "essence and dross decomposition" disorder and activate the function of the liver and its digestive system is adopted. Mongolian medicine modified Sugmul-7 is one of the commonly used Mongolian medicines in clinical practice. According to the etiology and pathogenesis of Mongolian medicine's "breast mass", the old professor has achieved long-term clinical use and has achieved good curative effects. Findings of research reports on the modern pharmacological action mechanism of this prescription.

Therefore, in this study, the proteomics was used to study the regulatory mechanism of modified sugmul-7 for the treatment of hyperplasia of the breast caused by endocrine disorders, which provided a scientific basis for the further development and clinical application of this prescription.

\section{Experimental Materials}

\subsection{Experimental Equipment}

YP-5002 electronic balance (Yuyao Jinnuo Electronic
Balance Equipment Co., Ltd.); SUNRISE microplate reader; TG16-WS ultracentrifuge (Xiangyi centrifuge company); HH-W600 constant temperature water bath (Jiangsu Jinyi Equipment Technology) Co., Ltd.); YDS-10B liquid nitrogen tank (Xi'an Huachen Lotte Lab Equipment Co., Ltd.); U410-86 ultra-freezing freezer (UK); $\mathrm{BCH} 198 \mathrm{~K}$ freezer (Haier Co., Ltd.); YB1201 electronic balance (Shanghai Haikang Equipment Co., Ltd.)

\subsection{The Main Reagents}

The main reagents 5-hydroxytryptamine 5-HT (batch number: 20181105, Shanghai Yuanye Biotechnology Co., Ltd.); cyclic guanosine cGMP (batch number: 20181105, Shanghai Yuanye Biotechnology Co., Ltd.); cyclic adenosine cAMP (Batch number: 20181105, Shanghai Yuanye Biotechnology Co., Ltd.; Triiodothyronine T3 (Lot number: 20181105, Shanghai Yuanye Biotechnology Co., Ltd.); Thyroxine T4 (Lot number: 20181105, Shanghai Yuanye Biotechnology Co., Ltd. Co., Ltd.); thyroid stimulating hormone TSH (batch number: 20181105, Shanghai Yuanye Biotechnology Co., Ltd.); gonadotropin releasing hormone GnRH (batch number: 20181105, Shanghai Yuanye Biotechnology Co., Ltd.);: 20181105, Shanghai Yuanye Biotechnology Co., Ltd.); RP C18 column; Pierce company TMT Mass Tagging Kits and Reagents kit; mass spectrometer is Thermo Scientific company Q Exactive.

Mass spectrometer; HPLC system is Diane NCS3500 system.

\subsection{The Experimental Drug}

The experimental drug modified Sugmul-7 (provided by the Mongolian Medicine Preparation Room of the Affiliated Hospital of Inner Mongolia University for Nationalities); estradiol (provided by Tianjin Jinyao Amino Acid Co., Ltd., standard: $1 \mathrm{mg} / \mathrm{ml}$, batch number: 201805211); Provided by Zhejiang Xianju Co., Ltd., standard: 1ml / 20mg, batch number: 201712001)

\subsection{Experimental Animal}

Experimental animals weighing $180 \sim 220 \mathrm{~g}$, SD rats (provided by Liaoning Changsheng Biotechnology Co., Ltd., batch number: SPF (Liao) 2018- 0002)

\section{Experimental Methods}

\subsection{Grouping and Dosage}

Ninety female SD rats were selected, and after being adapted for 2-4 days under experimental conditions, they were randomly divided into normal group, model group, and modified Sugmul-7 low, medium, and high doses in a total of 5 groups, 15 per group. After the start of the experiment, each rat except the normal group was intraperitoneally injected with estradiol $25 \mathrm{~d}$ and progesterone $6 \mathrm{~d}$ at a standard dose of $0.3 \mathrm{mg} / \mathrm{kg}$ for a total of 31 days. Each rat in the normal group was intraperitoneally injected with the same dose of $0.9 \%$ 
saline. Seven days after the start of model administration, each administration group began to infuse the corresponding dose of Mongolian medicine and flavored $0.5 \% \quad \mathrm{CMC}-\mathrm{Na}$ suspension of Sugmul-7 for a total of 25 days. The normal group and the model group were given the same dose of $0.5 \%$ CMC-Na solution. After the last administration, fasting and water for 24 hours, intraperitoneal injection with $10 \%$ chloral hydrate, anesthesia, blood extraction from abdominal aorta, centrifugation, separation of serum, storage at $-80^{\circ} \mathrm{C}$, and reserve.

\subsection{Determination of Indicators}

The serum 5-HT, cGMP, cAMP, T3, T4, TSH, GnRH, LH and other indicators were strictly operated and measured in accordance with the kit instructions.

\subsection{Proteomics}

The Q Exactive mass spectrometer from Thermo Scientific was used to identify and quantify proteins by secondary mass spectrometry.

\subsection{Statistical Methods}

The data of all measurement data of all indexes in serum were analyzed by SPSS17.0 statistical software. Two-sample isovariance and $\mathrm{T}$ test were used to analyze the significant differences in data between groups, $\mathrm{p}<0.05$ was considered statistically significant. The raw data of mass spectrometry were analyzed and analyzed by using the Sequest algorithm in the database of Proteome Discoverer software. Trypsin-specific digestion is used. A maximum of 2 missed sites are allowed. Cys iodide acetylation and TMT modification are fixed modification parameters. Methionine oxidation and phosphorylation are variable modification parameters. The tolerance is $15 \mathrm{ppm}$, and the product ion mass tolerance is $0.02 \mathrm{Da}$.

\section{Experimental Results}

\subsection{Mongolian Medicine Modified Sugmul-7 on Mass Spectral Analysis of breast Tissue Protein in Rats with Hyperplasia of the Breast}

\subsubsection{Differential Protein Clustering Results}

In this test, the number of proteins identified in the model group and the normal group was 6129 . The standard is 1.2 times 0.8333 times and the protein that satisfies $p<0.05$. The normal group and the model group have 132 proteins. There were 50 up-regulated and 82 down-regulated; compared with the administration group, there were 27 different proteins in the model group, of which 12 were up-regulated and 15 were down-regulated. The results are shown in Figure 1.

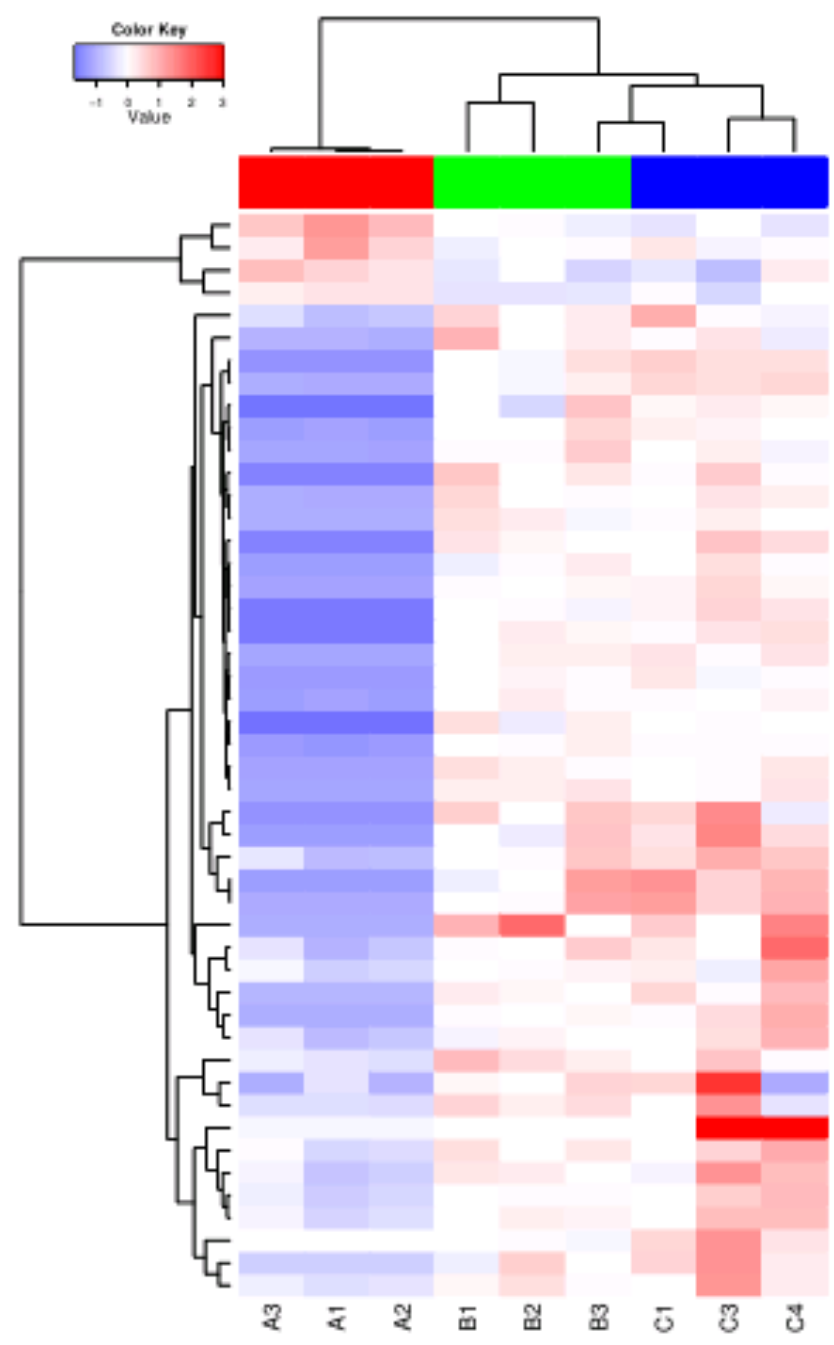

Figure 1. Differential protein clustering.

Note: A. Normal group

B. Model group

C. Administration group

\subsubsection{Enrichment Results-1.2-Fold Change and $p<0.05$ Differential Protein}

Based on the traditional Mongolian medicine theory and the pathogenesis of breast hyperplasia, 1.2 times to 0.8333 times as the standard, and the difference proteins and related websites with $\mathrm{p}<0.05$ are shown in Table 1 and the difference protein interaction network diagrams 1-10.

Table 1. LC / MS determination and stability of protein changes in breast hyperplasia rats.

\begin{tabular}{lllll}
\hline Accession & Gene_ID & Gene_Symbol & A-vs-B.FC & B-vs-C.FC \\
\hline NP_058738.1 & 24675 & Ppp3cb & 0.854351773608386 & 1.12651666958838 \\
NP_001004208.1 & 289144 & Cacybp & 0.906139145630226 & 1.09635553081322 \\
XP_006240461.1 & 681913 & Gstz1 & 1.14268977176518 & 1.07227190305282 \\
NP_001101152.1 & 310512 & Nmd3 & 0.895069492267815 & 1.20253710618143 \\
\hline
\end{tabular}

TMT10 plex after isotope labeling (A: normal group, B: model group, C: administration group) 


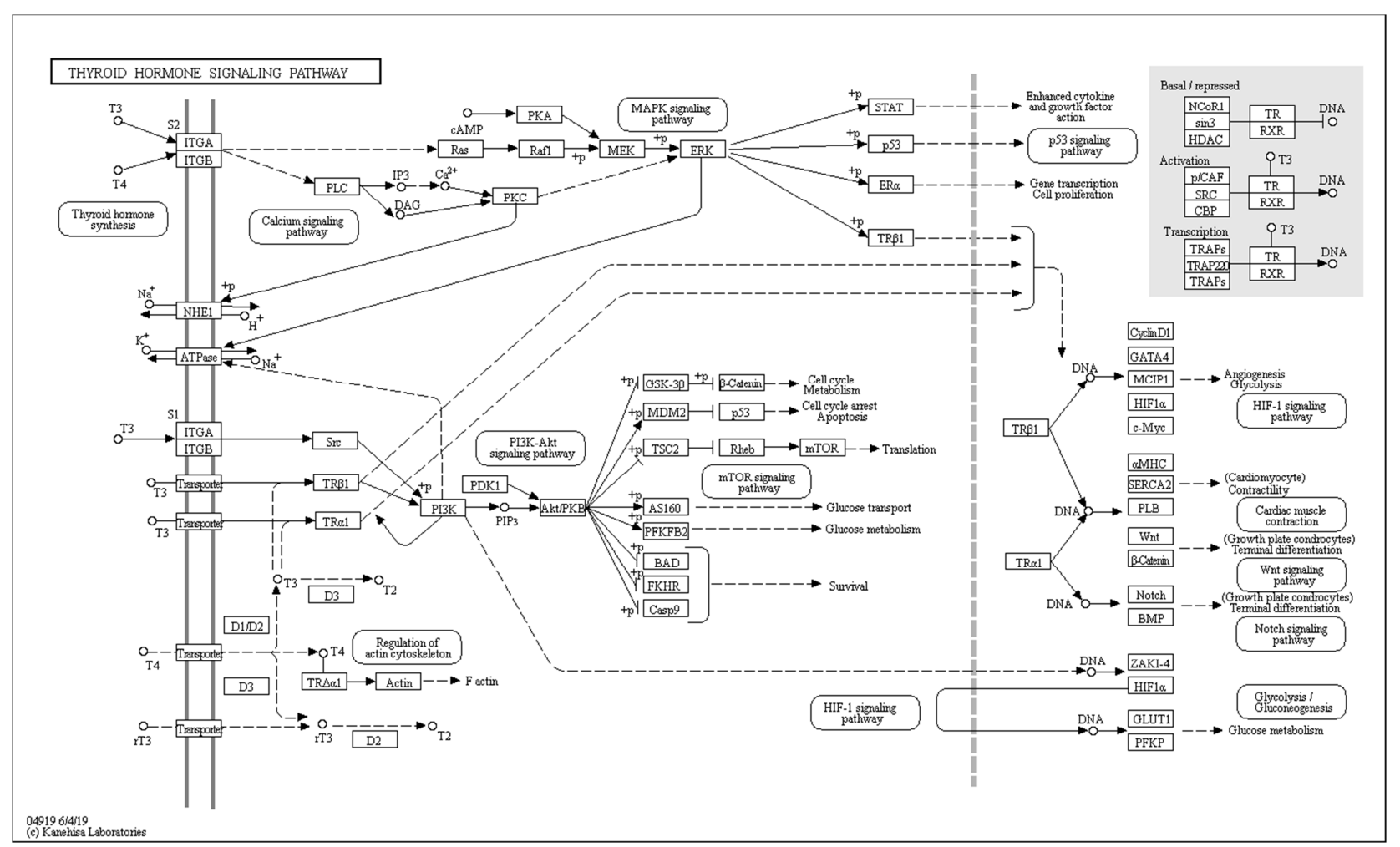

Figure 2. Map 04022 Pathway cGMP-PKG signaling.

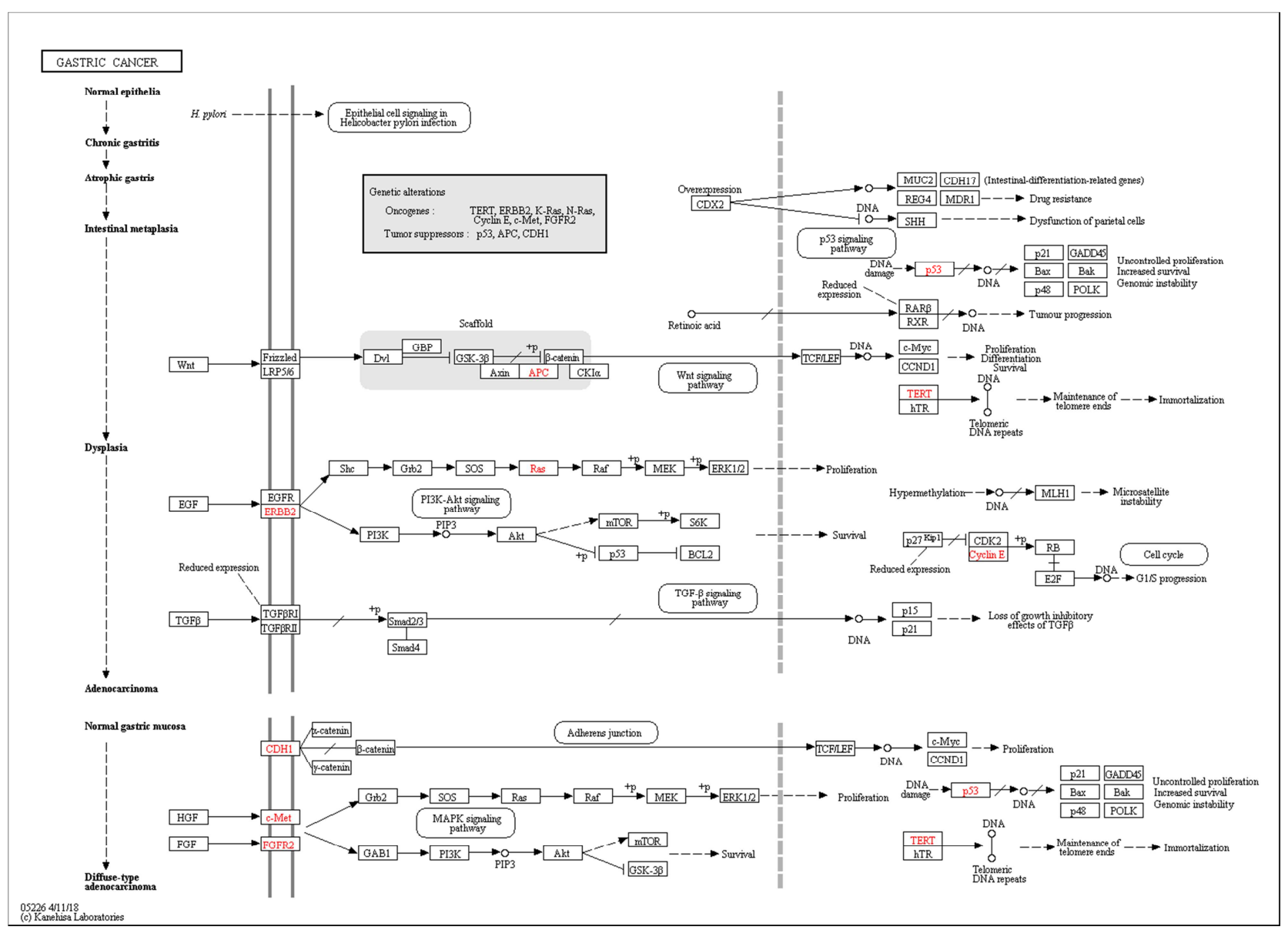

Figure 3. Map 04919 thyroid hormone signaling pathway. 


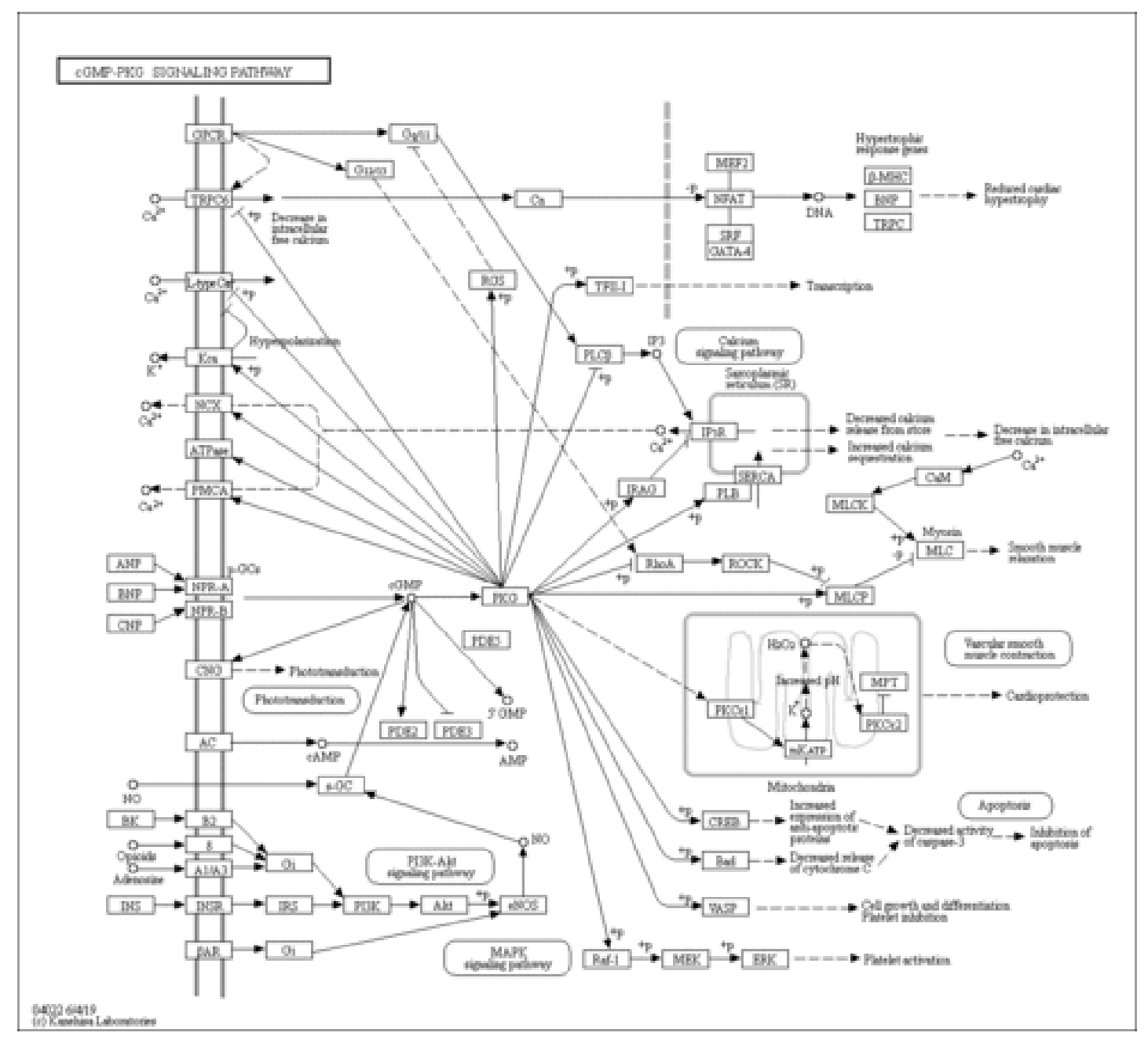

Figure 4. Map 05226 Path to the stomach cancer.

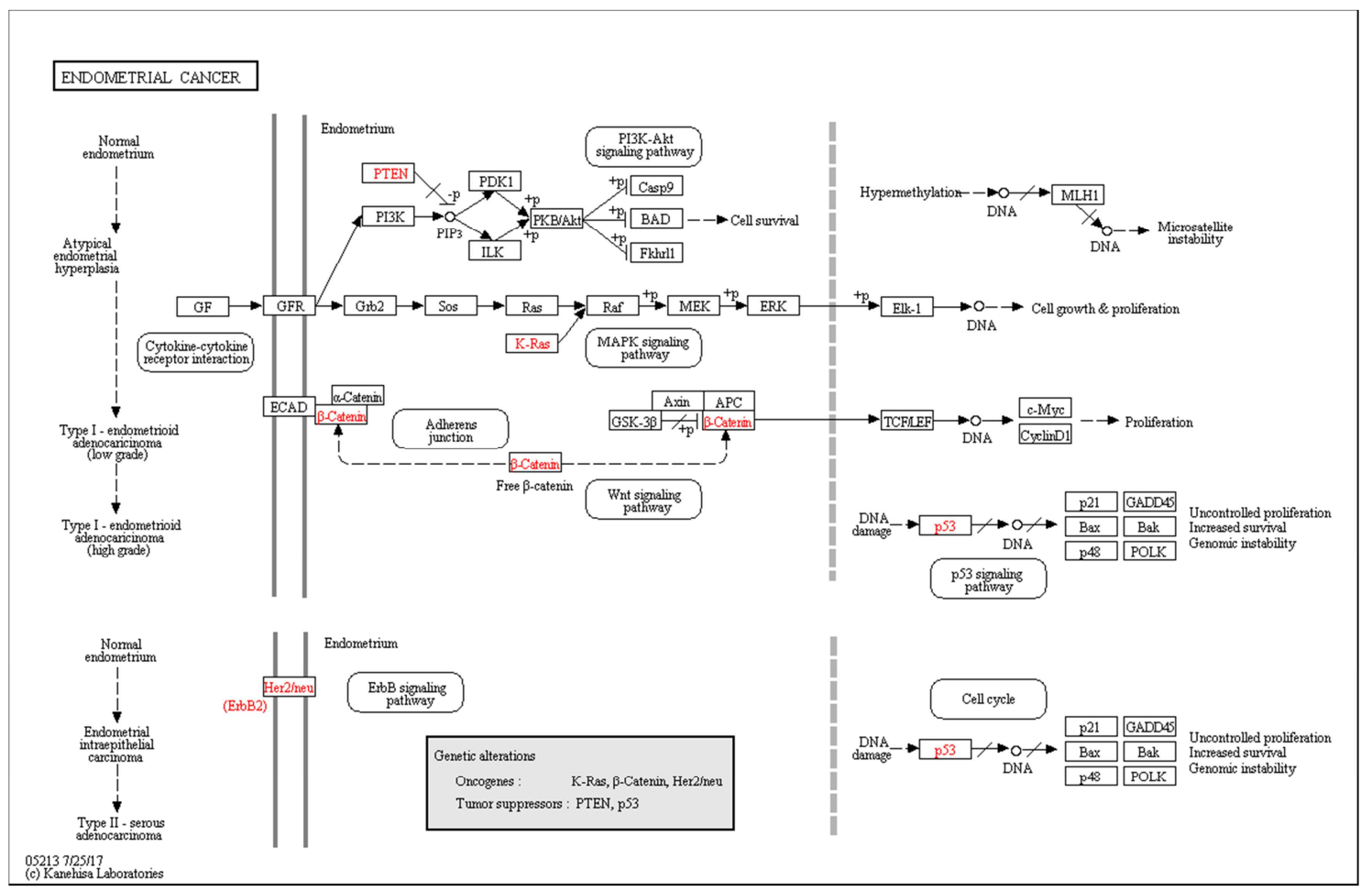

Figure 5. Map 05213 Route endometrial cancer. 


\subsection{Effect of Mongolian Medicine Modified Sugmul-7 on Serum cGMP and FSH of Rats with Hyperplasia of the Breast}

It is known from Table 2 that cGMP and FSH in the model group were significantly reduced, compared with the normal group (both $\mathrm{P}<0.05$ ) and cGMP was significantly increased in each administration group, compared with the model group ( $\mathrm{P}<0.05$ or $\mathrm{P}<0.01$ ). This was especially true for the middle and high dose groups which increased significantly, $(\mathrm{P}<0.05$ or $\mathrm{P}<0.01)$.

Table 2. Effect of Mongolian Medicine Modified Sugmul-7 on serum cyclic guanosine monophosphate (cGMP) and follicle stimulating hormone (FSH) of rats with hyperplasia of the breast.

\begin{tabular}{llll}
\hline Groups & Dose (g/kg) & cGMP (nmol/L) & FSH (IU/L) \\
\hline Normal & - & $3.5334 \pm 1.4084$ & $2.4232 \pm 0.2714$ \\
Model & - & $2.8404 \pm 0.2869^{*}$ & $2.2331 \pm 0.1245^{\#}$ \\
Sugmul-7 Low & 1.2 & $3.0799 \pm 0.2382^{\#}$ & $2.2427 \pm 0.1871$ \\
Medium & 2.4 & $3.2660 \pm 0.3843^{\# \#}$ & $2.3831 \pm 0.1614^{\#}$ \\
High & 3.6 & $3.0826 \pm 0.3586^{\#}$ & $2.6623 \pm 0.2934^{\# \#}$ \\
\hline
\end{tabular}

Note: * indicates a comparison with the normal group $\mathrm{P}<0.05$, \# and \#\# indicate a comparison with the model group $\mathrm{P}<0.05$, and $\mathrm{P}<0.01$ respectively.

\subsection{Effect of Mongolian Medicine Modified Sugmul-7 on Serum $E 2$ and LH in Rats with Hyperplasia of the Breast}

It is known from Table 3 that E2 in the model group was significantly increased and LH was significantly reduced, (P $<0.05$ or $\mathrm{P}<0.01)$ compared with the normal group and the $\mathrm{E} 2$ in each administration group was significantly reduced, (P $<0.01)$ compared with the model group. This was especially true, the middle and high dose groups increased significantly, $(\mathrm{P}<0.05$ or $\mathrm{P}<0.01)$.

Table 3. Effects of Mongolian Medicine Modified Sugmul-7 on serum Estradiol (E2) and Progesterone (LH) of rats with hyperplasia of breast $(n=15, X \pm S)$.

\begin{tabular}{llll}
\hline Groups & Dose $(\mathbf{g} / \mathbf{k g})$ & $\mathbf{E 2}(\mathbf{p m o l} / \mathbf{L})$ & LH $(\mathbf{m l u} / \mathbf{m l})$ \\
\hline Normal & - & $13.7685 \pm 1.4833$ & $10.4224 \pm 3.8431$ \\
Model & - & $15.2540 \pm 1.6569^{* *}$ & $8.0822 \pm 0.9935^{*}$ \\
Sugmul-7 Low & 1.2 & $10.6524 \pm 2.1495^{\# \#}$ & $8.5108 \pm 0.5721$ \\
Medium & 2.4 & $7.4337 \pm 0.7603^{\# \#}$ & $8.9364 \pm 1.0706^{\#}$ \\
High & 3.6 & $7.8677 \pm 1.9290^{\# \#}$ & $10.2203 \pm 1.3608^{\# \#}$ \\
\hline
\end{tabular}

Note: * and $* *$ indicate a comparison with the normal group $\mathrm{P}<0.05$ and $\mathrm{P}$ $<0.01$ respectively;

${ }^{\#}$ and ${ }^{\#}$ indicate a comparison with the model group $\mathrm{P}<0.05$, and $\mathrm{P}<0.01$ respectively.

\subsection{Effect of Mongolian Medicine Modified Sugmul-7 on Serum PRL and PROG in Rats with Hyperplasia of the Breast}

According to Table 4, the PRL of the model group was significantly increased and the PROG was significantly decreased, ( $\mathrm{P}<0.05$ or $\mathrm{P}<0.01)$ compared with the normal group; the PRL of each administration group was significantly reduced $(\mathrm{P}<0.01)$. The middle and high dose groups increased significantly, $(\mathrm{P}<0.01)$ compared with the model group.

Table 4. Effect of Mongolian Medicine Modified Sugmul-7 on serum prolactin (PRL) and progesterone (PROG) in rats with hyperplasia of the breast $(n=15, X \pm S)$

\begin{tabular}{llll}
\hline Groups & Dose $(\mathbf{g} / \mathbf{k g})$ & PRL $(\mathbf{n g} / \mathbf{m l})$ & PROG $(\mathbf{n g} / \mathbf{m l})$ \\
\hline Normal & - & $6.9125 \pm 0.5153$ & $2.5762 \pm 0.1711$ \\
Model & - & $7.6018 \pm 0.9280^{*}$ & $2.2619 \pm 0.2257^{* *}$ \\
Sugmul-7 Low & 1.2 & $6.1143 \pm 0.4860^{\# \#}$ & $2.1935 \pm 0.4398$ \\
Medium & 2.4 & $6.4661 \pm 0.7537^{\# \#}$ & $2.5899 \pm 0.1899^{\# \#}$ \\
High & 3.6 & $6.8029 \pm 0.9539^{\#}$ & $2.7726 \pm 0.5289^{\# \#}$ \\
\hline
\end{tabular}

Note: *and** indicate a comparison with the normal group $\mathrm{P}<0.05$ and $\mathrm{P}$ $<0.01$ respectively,

\# and ${ }^{\#}$ indicate a comparison with the model group $\mathrm{P}<0.05$, and $\mathrm{P}<0.01$ respectively.

\subsection{Effect of Mongolian Medicine Modified Sugmul-7 on Serum T4 and T3 of Rats with Hyperplasia of the Breast}

According to Table 5, T4 and T3 in the model group were significantly reduced, $(\mathrm{P}<0.05)$ compared with the normal group. The T4 and T3 in the high-dose group were significantly increased, $(\mathrm{P}<0.05)$ compared with the model group.

Table 5. Effects of Mongolian Medicine Modified Sugmul-7 on serum thyroxine (T4) and triiodothyronine (T3) of rats with hyperplasia of the breast $(n=15, X \pm S)$.

\begin{tabular}{llll}
\hline Groups & Dose $(\mathbf{g} / \mathbf{k g})$ & T4 $(\mathbf{n g} / \mathbf{m L})$ & T3 $(\mathbf{n g} / \mathbf{m L})$ \\
\hline Normal & - & $40.8959 \pm 9.5189$ & $2.0788 \pm 1.0758$ \\
Model & - & $34.4475 \pm 4.2636^{*}$ & $1.4374 \pm 0.1168^{*}$ \\
Sugmul-7 low & 1.2 & $31.8967 \pm 3.4947$ & $1.4055 \pm 0.1328$ \\
medium & 2.4 & $34.4971 \pm 4.8687$ & $1.3506 \pm 0.1761$ \\
high & 3.6 & $37.5134 \pm 4.3911^{\#}$ & $1.5955 \pm 0.2596^{\#}$ \\
\hline
\end{tabular}

Note: *\&** indicates a comparison with the normal group $\mathrm{P}<0.05$ and $\mathrm{P}$ $<0.01$ respectively,

\#and ${ }^{\# \#}$ indicate a comparison with the model group $\mathrm{P}<0.05$, and $\mathrm{P}<0.01$ respectively.

\subsection{Effect of Mongolian Medicine Modified Sugmul-7 on Serum GnRH and Testosterone of Rats with Hyperplasia of the Breast}

It is known from Table 6 that compared with the normal group, GnRH and $\mathrm{T}$ in the model group were significantly reduced, $\mathrm{P}<0.05$; compared with the model group, GnRH and $\mathrm{T}$ were significantly increased in the middle and high dose groups, $\mathrm{P}<0.05$ or $\mathrm{P}<0.01$.

Table 6. Effect of Mongolian Medicine Modified Sugmul-7 on Serum GnRH and testosterone of Rats with Hyperplasia of the breast $(n=15, X \pm S)$.

\begin{tabular}{llll}
\hline Groups & Dose (g/kg) & GnRH (mIu/ml) & T (pg/ml) \\
\hline Normal & - & $17.7599 \pm 1.8128$ & $1.8523 \pm 0.4784$ \\
Model & - & $16.2864 \pm 1.7028^{*}$ & $1.5139 \pm 0.0739^{*}$ \\
Low dose of Sugmul-7 & 1.2 & $16.3370 \pm 1.7800$ & $1.5753 \pm 0.1418$ \\
medium & 2.4 & $17.5747 \pm 1.3176^{\#}$ & $1.5970 \pm 0.1264^{\#}$ \\
high & 3.6 & $17.4522 \pm 1.4351^{\#}$ & $1.8368 \pm 0.2017^{\# \#}$ \\
\hline
\end{tabular}

Note: * means $\mathrm{P}<0.05$ compared with normal group,

${ }^{\#}$ and ${ }^{\# \#}$ mean $\mathrm{P}<0.05, \mathrm{P}<0.01$ compared with model group, respectively. 


\subsection{Effect of Mongolian Medicine Modified Sugmul-7 on Serum TSH and 5-HT in Rats with Hyperplasia of the Breast}

According to Table 7, TSH in the model group was significantly reduced, 5-HT was significantly increased, (P $<0.01$ respectively) compared with the normal group. The TSH in the high dose group was significantly increased, 5 -HT in the low dose groups was significantly reduced, compared with the model group ( $\mathrm{P}<0.05$ respectively).

Table 7. Effect of Mongolian Medicine Modified Sugmul-7 on Serum TSH and 5-HT in Rats with Hyperplasia of the breast $(n=15, X \pm S)$.

\begin{tabular}{llll}
\hline Groups & Dose $(\mathbf{g} / \mathbf{k g})$ & TSH $(\mathbf{m u} / \mathbf{l})$ & 5-HT $(\mathbf{n g} / \mathbf{m L})$ \\
\hline Normal & - & $3.7288 \pm 0.3243$ & $22.9170 \pm 2.4259$ \\
Model & - & $3.2169 \pm 0.3736^{* *}$ & $25.9204 \pm 2.0617^{* *}$ \\
Sugmul -7 low & 1.2 & $3.3136 \pm 0.5077$ & $23.6606 \pm 1.7594^{\#}$ \\
medium & 2.4 & $3.1568 \pm 0.4161$ & $24.9636 \pm 1.4455$ \\
high & 3.6 & $3.5995 \pm 0.5459^{\#}$ & $30.2254 \pm 2.2811$ \\
\hline
\end{tabular}

Note: *means $\mathrm{P}<0.05$ compared with normal group, \#means $\mathrm{P}<0.05$ compared with model group, \#\# means $\mathrm{P}<0.01$ compared with model group.

\section{Discussion}

Mongolian medicine modified Sugmul-7 is composed of Amomum kravanh Pierre ex Gagnep., Polygonatum sibiricum Delar. Ex Redoute, Panax ginseng C. A. Mey., Asparagus cochinchinensis (Lour.) Merr., Myristica fragrans Houtt., Eugenia caryophyllata Thunb., Aquilaria sinensis Lour, carthamus tinctorius $L$. The prescription function is suppressing Heyi, strengthening the body; treating heart and kidney Heyi disease, excessive leucorrhea, sore waist and legs, cold in the lower abdomen, irregular menstruation, weightlessness, etc. [9]. This prescription is a Mongolian medicine with long clinical experience based on the etiology and pathogenesis of the "breast mass" of Mongolian medicine. It has been used in the clinic for a long time and has achieved good therapeutic effects. No research report on the modern pharmacological action mechanism of this prescription has been found.

Drugs that regulate the function of
the liver and digestive system
( Amomum kravanh Pierre ex
Gagnep., Myristica fragrans
Houtt., Eugenia caryophyllata
Thunb., Aquilaria sinensis Lour,
carthamus tinctorius L.)
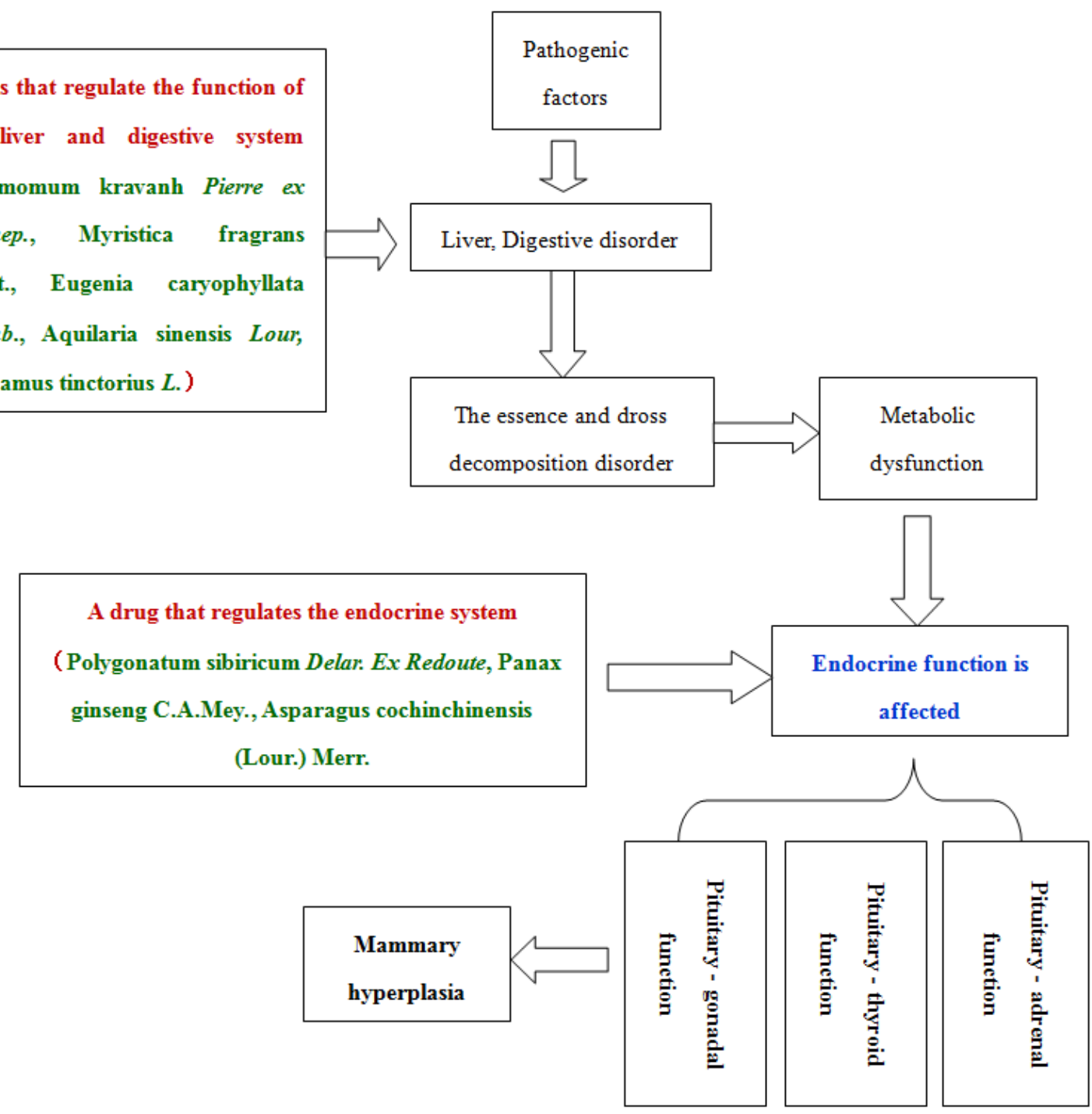

Figure 6. Mechanism for clinically treating hyperplasia of the breast, in Mongolian traditional medicine. 
According to modern research reports, Amomum kravanh contains volatile oil and trace elements such as $\mathrm{Ca}, \mathrm{Mn}$, and $\mathrm{K}$. It has the effects of anti-gastric ulcer, enhances gastric motility, and protects gastric mucosa [10]; Polygonatum, Ginseng, and Asparagus mainly contain polysaccharides. It has the function of improving immune function and regulating endocrine disorders [10]; safflower contains safflower yellow pigment, which can protect the liver and regulate the "essence and dross"; Myristica contains oily ingredients and has a sedative effect [10]; Eugenia are rich in the clove oil component has obvious sedative and hypnotic effects as well as protecting the gastric mucosa and anti-gastric ulcers [10]; Aquilaria sinensis contains volatile oils and has analgesic and antipyretic effects [10].

According to the above analysis, from the perspective of the composition of Mongolian medicine modified Sugmul-7, it consists of drugs that protect liver function, protect gastric mucosa, and drugs that regulate immune function and regulate endocrine function. Therefore, the formula's mechanism for clinically treating hyperplasia of mammary glands is likely to be to treat hyperplasia of the breast by regulating "endocrine function" disorders. As the picture shows.

Therefore, in this study, the proteomics method was used to find the differential protein of Mongolian medicine modified Sugmul-7 for the treatment of breast hyperplasia, and to explain the endocrine regulation mechanism of the prescription for the treatment of breast hyperplasia.

According to the results of this experiment, based on the traditional theory of Mongolian medicine and the pathogenesis of breast hyperplasia, 1.2 times to 0.8333 times are used as the standard. Four differential proteins were found, which are Ppp3cb, Cacybp, Gstz1Gstz1, and Nmd3. A total of 10 related pathways were found.

Among them (1) cGMP-PKG signaling pathway [11]: Cyclic GMP is the second messenger in the cell, mediating the effects of nitric oxide and natriuretic peptide, and regulating a wide range of physiological processes. Increased intracellular cGMP levels exert their physiological roles through two forms of cGMP-dependent protein kinase (PKG), cGMP-regulated phosphodiesterase (PDE2, PDE3), and cGMP-gated cation channels, of which PKGs may be the main medium. The PKG1 subtype-specific activation of established substrates leads to a decrease in cytosolic calcium concentration and / or a decrease in the sensitivity of myofilaments to $\mathrm{Ca}^{2+}\left(\mathrm{Ca}^{2+}\right.$-desensitization), leading to smooth muscle relaxation. The results of this test showed that cGMP was significantly reduced in the normal group compared with the model group. This may be the mechanism of calcium loss caused by clinically induced endocrine disorders in females caused by decreased calcium levels in the body's myofilaments and bone, significantly increased. Mongolian medicine modified Sugmul-7 is one of the mechanisms of regulating calcium loss caused by endocrine disorders. The results of this experiment are shown.

(2) Thyroid hormone signaling pathway [12]: Thyroid hormones (THs) are important regulators of growth, development and metabolism. The effect of thyroid hormone is mainly mediated by T3 (3,5,3'-triiodo-1-thyrosine). Thyroid hormones, levothyroxine (T4), and T3 enter cells through transporters. Although the main form of thyroid hormone in the blood is $\mathrm{T} 4$, it is converted intracellularly to the more active hormone T3. T3 binds to nuclear thyroid hormone receptors (TRs), which act as ligand-dependent transcription factors and control the expression of target genes (genomic effects). Non-genetic mechanisms begin at the integrin receptor. The plasma membrane $\alpha$ (v) $\beta$ (3) -integrin has different binding sites for $\mathrm{T} 3$ and T4. One binding site only binds $\mathrm{T} 3$ and activates the phosphatidylinositol 3-kinase (PI3K) pathway. The other binding site binds T3 and T4 and activates the ERK1 / 2 MAP kinase pathway. The results of this experiment show that $\mathrm{T} 3$ and $\mathrm{T} 4$ are decreased in the model group to compare normal group, and T3 and T4 are significantly increased in the high-dose group to compare the model group.

(3) Estrogen signaling pathway [13]: Estrogen is a steroid hormone that regulates excessive physiological processes in mammals, including reproduction, cardiovascular protection, bone integrity, intracellular environment stability, and behavior. Estrogen regulates its cellular effects through two signaling pathways, which are divided into "nuclear-induced steroid signaling" and "membrane-induced steroid signaling." In the "nuclear" pathway, estrogen binds to ER alpha or ER beta, which in turn is translocated to the nucleus, binds to DNA on the ERE element, and activates ERE-dependent gene expression. In the "membrane" pathway, estrogen can function through a subpopulation of estrogen receptors on the plasma membrane or new $G$ protein-coupled E2 receptors (GPER). After activating these receptors, various signaling pathways (i.e., $\mathrm{Ca}^{2+}$, cAMP, and protein kinase cascades) are rapidly activated and eventually affect downstream transcription factors. Studies have shown that this pathway causes abnormalities in breast cancer. The results of this experiment show that the normal group is significantly higher than the model group, and each administration group is significantly lower than the model group.

(4) Gastrointestinal signal-related pathways [14]: Gastric cancer is one of the most common cancers in the world. According to Lauren's histological classification, gastric cancer is divided into two different histological types-intestinal and diffuse. Several genetic changes have been identified in intestinal gas chromatography. Intestinal metaplasia is characterized by mutations in the p53 gene, reduced expression of retinoic acid receptor beta (RAR $\beta)$, and reduced expression of telomerase reverse transcriptase. Gastric adenomas further showed APC gene mutations, decreased p27 expression, and cyclin E amplification. In addition, in more advanced gas chromatography, amplification and over expression of c-ErbB2, reduced expression of TGF- $\beta$ receptor $i$-type, and complete loss of p27 expression are usually observed. The main molecular changes observed in diffuse GCs include CDH1 mutations 
and loss of E-cadherin function due to MET and FGFR2F amplification.

(5) Reproductive system-related pathways [15]. Endometrial cancer is the most common gynecological malignancy and the fourth largest malignancy after breast cancer, colorectal cancer, and lung cancer among women in developed countries. The two endometrial cancers are different biologically and clinically. Type uterine cancer is associated with endometrial hyperplasia, frequent expression of estrogen and progesterone receptors, and younger age, while type uterine cancer has nothing to do with estrogen, and is frequently associated with atrophic endometrium, estrogen, and progesterone receptors. Lack is related to older age. The morphological differences of these cancers are reflected in their molecular genetic maps. The $\mathrm{i}$ type shows DNA mismatch repair and mutation defects in PTEN, K-ras and $\beta$-catenin, and the ii type shows aneuploidy, p53 mutations, her2 / neu amplification.

In summary, the above pathways are related to the treatment of hyperplasia of the breast caused by endocrine disorders with Mongolian medicine modified Sugmul-7, which is completed by the gastrointestinal and endocrine regulating functions.

\section{Conclusion}

In conclusion, the above pathways are all related to the treatment of breast hyperplasia caused by endocrine disorders with Mongolian medicine sugmul-7, which is completed through the regulation of gastrointestinal tract and endocrine.

\section{Acknowledgements}

I would like to express my special thanks of graduate to my teacher Tsend-Ayush as well as our research team. This research project was funded from affiliated hospital of Inner Mongolia University for nationalities which named Research of Sugmul-7+ safflower on experimental "breast mass" and mechanism of hyperplasia of the breast.

\section{References}

[1] Z. Ying, H. Wenshan, Wang. Wei, et al. (2009). Screening and epidemiological factors of 75,490 women with breast diseases. Journal of Chinese women's health, 24: 1465-1466.

[2] H. Kefei, G. Qi, P. Xiao Hua et al. (2013). Clinical study on influencing factors of 435 cases of breast hyperplasia. Chinese journal of experimental formulations. 19 (9): 319-322. (in Chinese with English abstract).

[3] Fan F. Ying. (2002). Shallowly talk about the maladjustment and Mammary gland hyperplasia. Journal of Nanjing University of Chinese medicine. 18 (5): 266-267. (in Chinese with English abstract).

[4] Lu Jingbo, Qiu Guifang. (2003) Diagnosis and treatment of 10 cases of Hyperprotinemia. Journal of Medical theory and practice. 16 (1): 56-57.

[5] W. Jieping, Q, Fazu, H. Jiaxiun. Surgery. Beijing: People's medical publishing house, 2002: 829-830. (in Chinese).

[6] Zhao W J, Wang J W, Chang J Z, et al. (2014) Effect of Xianlu Xiaopi capsule on expression of VEGF, bFGF and MVD in breast hyperplasia mice. Shanghai Journal of Traditional Chinese medicine. 48 (3): 74. (in Chinese with English abstract).

[7] Lin Yi, tang hanjun. Modern Chinese medicine on breast disease [M]. Beijing: people's medical publishing house, 2003, 5: 103 .

[8] Wang zege. Mongolian medicine and surgery. Hohhot: Inner Mongolia people's publishing house, 1986, 12: 287.

[9] Editorial committee of encyclopedia of Chinese medicine. Encyclopedia of Chinese medicine, Mongolian medicine. Shanghai: Shanghai science and technology press, 1992: 274.

[10] Wang X L, Pharmacology of Mongolian medicine. Hohhot: Inner Mongolia people's publishing house, 2006, 6: 36, 176, $33,28$.

[11] Signaling for contraction and relaxation in smooth muscle of the gut. Annu Rev Physiol 68: 345-74 (2006) DOI: 10.1146/annurev.physiol.68.040504.094707.

[12] Membrane receptor for thyroid hormone: Physiologic and pharmacologic implications. Annu Rev Pharmacol Toxicol 51: 99-115 (2011) DOI: 10.1146/annurev-pharmtox-010510-100512.

[13] Estrogen signaling in the Hypothalamus. Vitam Horm 71: 123-45 (2005) DOI: 10.1016/S0083-6729(05)71005-0.

[14] From inflammation to gastric cancer - the importance of Hedgehog/GLI signaling in Helicobacter pylori-induced chronic inflammatory and neoplastic diseases. Cell Commun Signal 15: 15 (2017) DOI: 10.1186/s12964-017-0171-4.

[15] Molecular genetic pathways in various types of endometrial carcinoma: from a phenotypical to a molecular-based classification. Virchows Arch 444: 213-23 (2004) DOI: 10.1007/s00428-003-0947-3. 